\title{
AN EXAMINATION OF THE EFFECT OF ARRAY WEIGHTING FUNCTION ON RADAR TARGET DETECTABILITY
}

\author{
C.M. Alabaster*, E.J. Hughes* \\ *Cranfield University, Shrivenham, UK. Email c.m.alabaster@cranfield.ac.uk
}

Keywords: radar detection, clutter, medium PRF, weighting function, antenna, airborne radar.

\begin{abstract}
This paper describes a methodology to assess the detectability of targets by an airborne fire control radar operating in a medium PRF mode in the presence of strong ground clutter as a function of the transmitting and receiving antenna array weighting functions and proportion of failed array elements. It describes the radar, antenna and clutter modelling processes and the method by which target detectability is quantified. The detectability of targets in clutter is described using a detectability map, which provides a useful means of comparing target detectability as clutter conditions change. It concludes that the best target detectability is to be achieved using those weighting functions on transmit and receive which result in the lowest average sidelobe levels but that the margins between the more highly tapered weighting functions were small.
\end{abstract}


Furthermore, it concludes that target detectability degrades as the proportion of failed elements increases. A failure of $5 \%$ of the elements gave modest, though meaningful, degradations in target detectability and would therefore form a suitable upper limit.

\section{Introduction}

This paper describes simulation work to assess the detectability of targets by an airborne fire control radar (FCR) operating in a medium pulse repetition frequency (PRF) mode in the presence of strong ground clutter as a function of transmitting and receiving array weighting functions. This paper also models the compromise on target detectability through the graceful degradation of up to $5 \%$ of failed array elements. It describes the radar, antenna and clutter modelling for a system operating a 3 of 8 medium PRF schedule waveform. Medium PRF waveforms and the selection of PRFs are described in the authors' previous papers $[1,2,3,4,5]$.

Target detectability depends on the number of PRFs in which any target is visible and on the probability of detection $\left(\mathrm{P}_{\mathrm{d}}\right)$ in each PRF [6]. The $\mathrm{P}_{\mathrm{d}}$ in each PRF is determined by the signal to noise plus clutter ratio (SNCR), amongst other factors, and varies across the range and velocity (Doppler) detection space of the radar due to the ambiguous repetition of clutter across this detection space. Minimizing side lobe clutter (SLC) through the minimization of antenna sidelobe level is a design priority for such systems. This may be achieved by applying a tapered illumination function across the antenna aperture and can be implemented readily by appropriate amplitude and phase weightings of the elements of an active electronically scanned array (AESA) antenna. However, tapered illumination functions result in a reduction in main beam boresight gain together with a broadening of the main beam, both of 
which are further degraded when the beam is phase steered away from its mechanical boresight. Furthermore, phase steering tends to generate increased sidelobes. Thus there appears to be a conflict of interests in applying tapered illumination across an array antenna as far as target detection is concerned; on the one hand the tapered illumination reduces the sidelobe level but on the other it leads to a loss of main beam gain. Thus both clutter and target signal strengths are reduced through the use of a tapered antenna illumination or, conversely, both are maximized for a uniformly illuminated antenna. The question arises as to whether tapered illumination actually leads to increased target detectability or not in scenarios in which target detection is likely to be clutter limited (i.e. low flying, look-down).

This question has been addressed by modelling the clutter scene in an airborne FCR for various combinations of transmitting and receiving array weighting functions, azimuth and elevation steering angles, platform altitudes and probabilities of failed array elements. For each combination of conditions, target detectability is derived over the full range/velocity detection space of the radar. Comparisons between the target detectability of the various conditions are evaluated in order to determine the optimum transmitting and receiving array weighting functions. As a secondary aim, the degradations resulting from a loss of up to $5 \%$ of the array elements were also modelled and quantified.

Section 2 of this paper describes the radar, antenna and clutter modelling processes and the method by which target detectability is derived and compared. In section 3 , the results are presented and discussed. Finally, section 4 draws conclusions. 


\section{Modelling processes}

\subsection{Radar model}

The radar model is intended to be representative of a modern FCR. It has been assumed that the radar operates on a medium PRF schedule of 8 PRFs and requiring target data in a minimum of 3 PRFs for ambiguity resolution. It is further assumed that range and Doppler ambiguities are resolved using the coincidence algorithm. The selection of the 8 pulse repetition intervals $(\mathrm{PRI}=1 / \mathrm{PRF})$ was made in a separate exercise as described in $[1,2,3,4,5]$. It is commonplace to use a filter to reject main beam clutter (MBC) over a narrow bandwidth in the Doppler domain prior to FFT processing. It is also commonplace to apply platform motion compensation (PMC) such that the velocity of mainbeam boresight detections are ground referenced. In this way, MBC is centred at zero Doppler and at multiples of the PRF. PMC is assumed in this study, however, no $\mathrm{MBC}$ filtering is assumed. This ensures that target detectability may be evaluated even in regions of strong MBC. The radar platform altitudes considered were $1000 \mathrm{~m}$ and $5000 \mathrm{~m}$ and the platform velocity was taken as $300 \mathrm{~ms}^{-1}$. Other parameters of the radar model are summarised in Table 1. 


\begin{tabular}{|l|l|}
\hline Parameter & Value \\
\hline Frequency & $10 \mathrm{GHz}$ - fixed \\
PRI (=1/PRF) & $35.5,38.5,44.5,49.5,56.0$, \\
Space charging time & $64.5,69.0$ and $94.0 \mu \mathrm{s}$ \\
Target illumination time & $42.5 \mathrm{~ms}$ \\
Duty ratio & $10 \%($ fixed $)$ \\
(Transmitted pulse width & $10 \%$ of PRI $)$ \\
Peak transmitted power & $10 \mathrm{~kW}$ \\
Pulse compression & Yes - variable with PRF \\
Range resolution & $75 \mathrm{~m}(0.5 \mu \mathrm{s}$ in time $)-$ fixed \\
Doppler processing & 64 point FFT \\
Eclipsing blindness & transmitted pulse width $+0.5 \mu \mathrm{s}$ \\
Maximum range & $185 \mathrm{~km}(100 \mathrm{nmi})$ \\
\hline Saximum velocity & $500 \mathrm{~ms}($ Doppler $=100 \mathrm{kHz})$ \\
\hline
\end{tabular}

Table 1: Radar Model Parameters

\subsection{Antenna model}

A planar AESA antenna comprising 1041 elements distributed in a diamond lattice over a circular area of nominal diameter $56 \mathrm{~cm}$ was modelled. The element spacings were nominally a half wavelength. Three possible transmitting array weighting 
functions were considered: Uniform, Radial Transmit Taper (RTT) [7,8] and Successive Projection Transmit Nulling (SPTN) [8,9] and two possible receiving array weighting functions were considered: Taylor $35 \mathrm{~dB}$ and Taylor $45 \mathrm{~dB}(\bar{n}=2)$. In addition to these 6 combinations of weighting functions, a seventh, that of Uniform on transmit and Uniform on receive, was also considered for comparative purposes. The 7 combinations of the transmitting and receiving array weighting functions (named patterns) are defined in Table 2.

\begin{tabular}{|c|c|c|}
\hline patterns & Transmit Weighting Function & Receive Weighting Function \\
\hline 1 & Uniform & Uniform \\
2 & Uniform & Taylor $35 \mathrm{~dB}$ \\
3 & Uniform & Taylor $45 \mathrm{~dB}$ \\
4 & RTT & Taylor $35 \mathrm{~dB}$ \\
5 & RTT & Taylor $45 \mathrm{~dB}$ \\
6 & SPTN & Taylor $35 \mathrm{~dB}$ \\
7 & SPTN & Taylor $45 \mathrm{~dB}$ \\
\hline
\end{tabular}

Table 2: Combinations of Array Weighting Functions

The weighting function data defined the magnitude and phase of the current exciting each element. Furthermore, each element of the array was defined as having a power gain pattern which varies as the cosine of the angle off the mechanical boresight. The phase of each element was under the control of a 6 bit phase shifter. The magnitude 
(power) of each element was subject to a tolerance of $0.3 \mathrm{~dB}$ (Gaussian of zero mean and $\sigma=0.3 \mathrm{~dB}$ ) and a phase tolerance of $2^{0}$ (Gaussian of zero mean and $\sigma=2^{0}$ ). It was also necessary to account for the random failure of $0 \%, 2 \%$ and $5 \%$ of the elements. This was modelled by including a function which set the probability of each element having zero amplitude to $0.00,0.02$ and 0.05 , respectively. This ensured that the selection of failed elements was randomized but that each element was equally likely to fail. The complete loss of elements i.e. zero transmitted and received power, was the only failure mode considered in this study since it is the most damaging to the array radiation pattern. The authors acknowledge the possibility of a host of other possible failures (e.g. partial loss of powers, loss in either transmit or receive modes and increased receiver noise figure) but these were considered outside the scope of this study. Notwithstanding this, it would be easy to accommodate such failure modes within the model. The simulation has been conducted in MATLAB; it loads in element amplitude and phase data from a large data file matrix and results in the antenna gain being expressed as a large two-dimensional array. It would be possible to overwrite specific elements of the array (i.e. specific elements of the array data matrix) to adjust their amplitude and/or phase responses, as required. The organisation of such matrices also enables whole rows, columns or sub-arrays to be readily adjusted, as required. Simple phase gradients were derived which provided the necessary steering in both azimuth and elevation. Simulations were run for azimuth steering angles of $0^{\circ}$ (dead ahead), $30^{\circ}$ and $56^{\circ}$ and for elevation angles of $0^{\circ}$ (towards horizon, both platform altitudes) and $5.5^{0}$ down (5000m altitude only). Therefore, there were a total of 9 combinations of altitude, azimuth and elevation steering angles together with 3 probabilities of failed elements giving rise to 27 differing conditions for each of the 7 patterns i.e. 189 total simulations. 
The far-field radiation pattern was derived by computing the two-dimensional Fourier transform over the array surface. Only the lower hemisphere need be derived since only this portion illuminates the ground. Furthermore, only the forward looking halfhemisphere was considered since the rear-ward looking pattern is likely to be dominated by the interaction with the radome which was outside the scope of this study. The rear-ward pattern results in negligibly low levels of clutter in the negative Doppler domain. Later analysis (section 2.5) supports this assumption; results are dominated by the far higher antenna gains in the forward half-hemisphere.

Each element produces 10 Watts of RF power giving rise to approximately $10 \mathrm{~kW}$ of total power. The peak main beam boresight gain for the uniform weighting function (assuming no magnitude and phase errors and zero failed elements) was normalised to 33.5dBi by an appropriate scaling factor. All other radiation diagrams were scaled by the same factor to ensure that the computed radiation diagrams represented the true effective radiated power (ERP). Example radiation diagrams are reproduced in Figures 1 and 2. Figure 1 illustrates the best case radiation diagram of the Uniform weighting function having zero phase steering angles, no magnitude and phase errors and zero failed elements, whereas Figure 2 illustrates the worst case radiation diagram of the Uniform weighting function having phase steering angles of $-5.5^{0}$ in elevation and $56^{\circ}$ in azimuth, $0.3 \mathrm{~dB}$ magnitude and $2^{0}$ phase errors and $5 \%$ failed elements. 


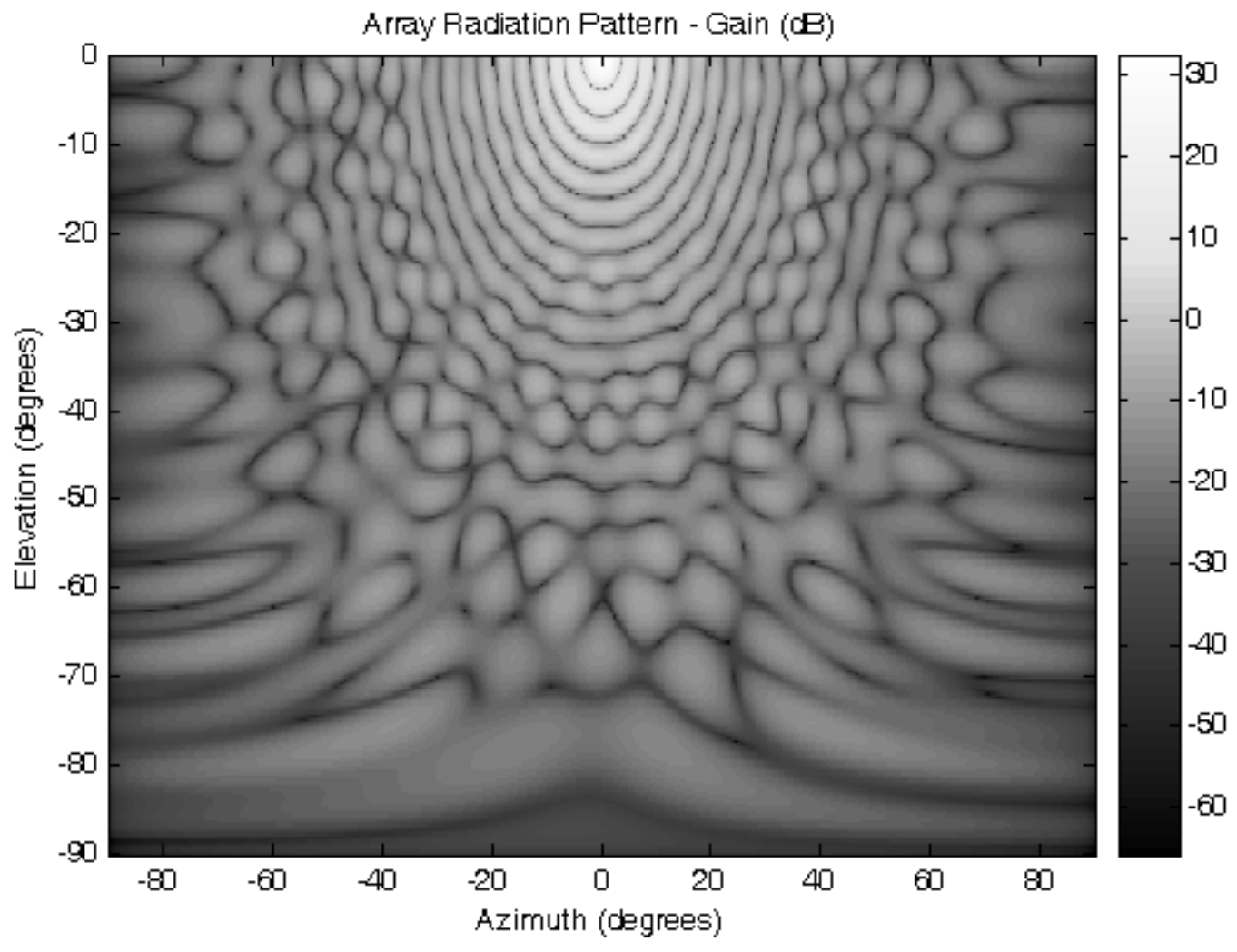

Figure 1: Ideal Array for Uniform Weighting Function

(no steering angle, zero magnitude and phase errors, zero failed elements) 


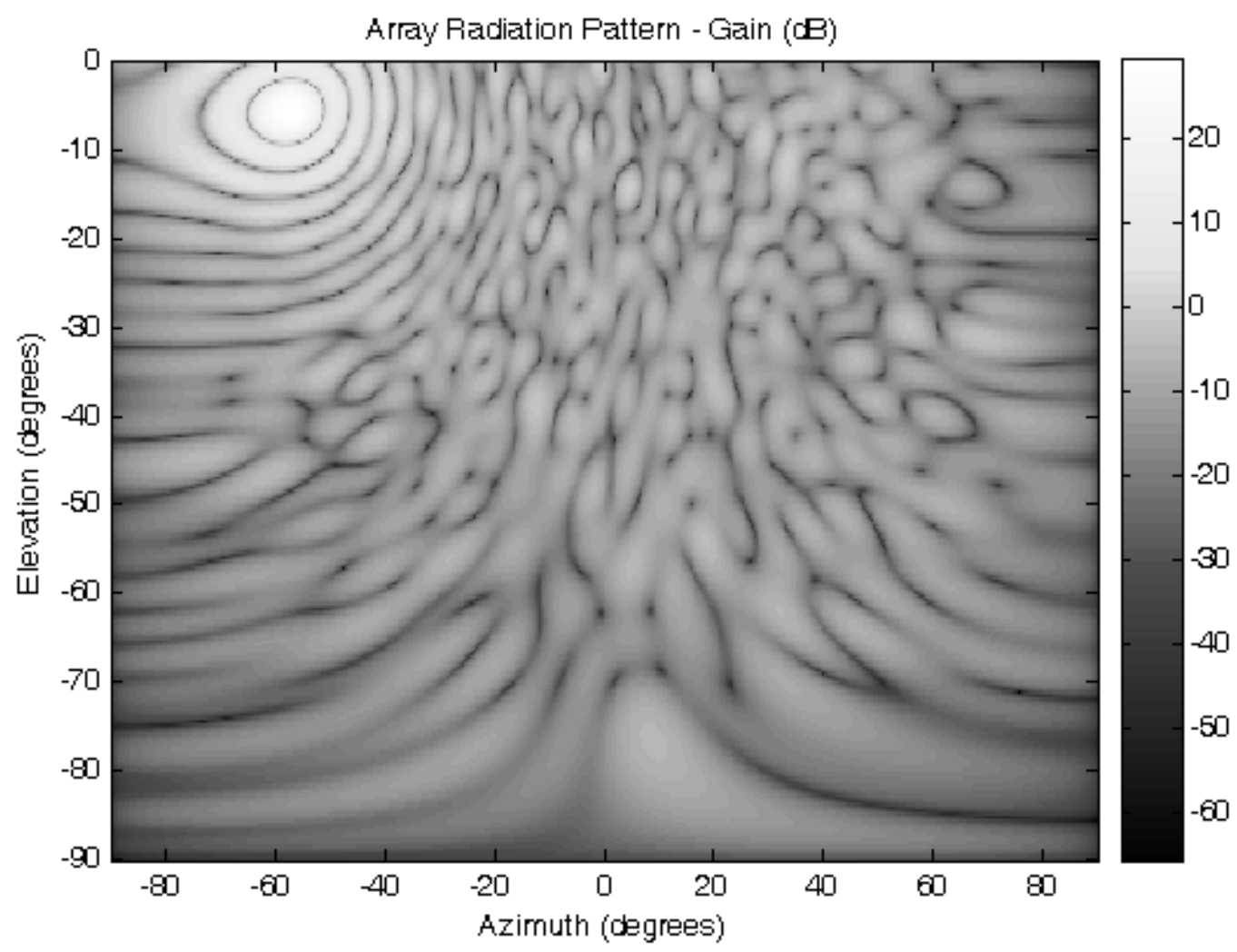

Figure 2: Worst Case Array for Uniform Weighting Function

$\left(56^{0}\right.$ azimuth and $-5.5^{0}$ elevation steering angles, $0.3 \mathrm{~dB}$ magnitude and $2^{0}$ phase errors, 5\% failed elements)

The radiation pattern of the ideal antenna uniformly weighted illustrated in Figure 1 indicates a main beam boresight gain of $33.53 \mathrm{~dB}$, a $3 \mathrm{~dB}$ beamwidth of $3.08^{0}$ and a peak sidelobe level (SLL) of $17.50 \mathrm{~dB}$ below the main beam. When elemental magnitude and phase tolerances of $0.3 \mathrm{~dB}$ and $2^{0}$, respectively, and a proportion of failed elements of 5\% are all applied, the changes in these parameters are barely noticeable. However, when the beam is steered well away from its mechanical boresight, as in Figure 2, the antenna parameters degrade to the following: main beam boresight gain $=30.21 \mathrm{~dB}$, peak SLL $=-16.86 \mathrm{~dB}$ and azimuth $3 \mathrm{~dB}$ beamwidth $=5.71^{0}$. Similar plots affirm the peak SLL of $-35 \mathrm{~dB}$ for the Taylor $35 \mathrm{~dB}$ function. However, the Taylor $45 \mathrm{~dB}$ function results in a peak SLL of around $-43 \mathrm{~dB}$ which is slightly 
above the expected level of $-45 \mathrm{~dB}$. This discrepancy is due to the magnitude and phase tolerances of each element, since when these were set to zero, a peak SLL of $44 \mathrm{~dB}$ was obtained. The radiation patterns for the radial transmit taper (RTT) and successive projection transmit nulling (SPTN) functions indicate that the RTT has a maximum SLL of around $-20.6 \mathrm{~dB}$, whereas the SPTN has a maximum SLL of around $-16.7 \mathrm{~dB}$, although its sidelobes at large angular offsets from the mainbeam boresight decay away more quickly than for the RTT. The SPTN function also gives rise to unusually large sidelobes some $11.5^{0}$ below the mainbeam boresight; these are at a level of $-23.0 \mathrm{~dB}$. A summary of the radiation patterns for each array weighting function is given at Table 3 under the ideal cases of zero magnitude/phase errors, zero failed elements and zero steering angles. Note that the SPTN pattern has been optimised to reduce lower hemisphere sidelobes at the expense of larger sidelobes in the upper hemisphere. The authors acknowledge that sub-array processing and radome effects may limit the integrity to which these idealised patterns may be reproduced, however, the use of idealised radiation patterns in this work is sufficient to demonstrate the principle. 


\begin{tabular}{|l|l|l|l|l|l|}
\hline Weighting Function & Peak & Gain Loss & 3dB & Peak & rms \\
& Gain & wrt Uniform & beamwidth & SLL & SSL \\
& $(\mathrm{dBi})$ & $(\mathrm{dB})$ & $(\mathrm{deg})$ & $(\mathrm{dB})$ & $(\mathrm{dB})$ \\
\hline Uniform & 33.53 & 0 & 3.08 & -17.50 & -32.12 \\
\hline Taylor 35dB & 32.41 & 1.111 & 3.74 & -34.82 & -47.20 \\
\hline Taylor 45dB & 31.70 & 1.822 & 4.04 & -43.63 & -54.82 \\
\hline RTT & 32.99 & 0.538 & 3.46 & -20.63 & -35.13 \\
\hline SPTN & 33.30 & 0.228 & 3.33 & -16.68 & -32.73 \\
(lower hemisphere) & & & & $(-18.86)$ & $(-35.90)$ \\
\hline
\end{tabular}

Table 3: Summary of Array Radiation Patterns

\subsection{Clutter model}

In modelling the clutter and noise all statistical variation has been eliminated in order to permit small changes in target detectability to be resolved. Clutter modelling in the forward hemisphere only is required. The method previously described in [1] is used. Clutter is mapped by considering the surface under the radar to be marked out by a grid along orthogonal $x$ and $y$ coordinates centred at 0,0 directly under the radar. The model steps through increments in the $x$ and $y$ coordinates in the forward half space (i.e. positive $y$ ) out to the maximum range of interest. At each location the model computes the slant and ground ranges and the resolved Doppler shift along the line of sight to the radar together with the grazing angle, clutter backscatter coefficient and clutter radar cross section (RCS). An important aspect of the clutter mapping process 
is the resolution of the increments along the $x / y$ coordinate system. At each location the clutter RCS is computed on the basis of a clutter area equal to the square of the $x / y$ resolution. Ideally, the $x / y$ resolution should be finer than the radar range resolution since otherwise there will be large clutter patches appearing in some resolution cells and nothing in neighbouring cells. However, very fine $x / y$ resolution is unnecessary and increases the computational time. Since the radar range resolution is 75 metres an $x / y$ resolution of 50 metres has been used.

The clutter backscatter coefficient (BSC) is a function of the grazing angle, $\theta_{g}$, which is computed for each point in the clutter modelling process. The BSC was defined as:

$$
B S C=\sigma_{0} \sin \left(\theta_{g}\right)+\sigma_{0 V} \exp \left(\frac{-\left(90-\theta_{g}\right)}{\theta}\right)
$$

where $\sigma_{0}=-15 \mathrm{dBm}^{2}$ and $\sigma_{0 V}=-5 \mathrm{dBm}^{2}$ (in linear units),

$$
\text { and } \theta=\frac{-20}{\log _{e}\left(\sin 70^{\circ} / A\right)} \quad \text { and } A=\frac{\sigma_{0 V}}{\sigma_{0}}
$$

$\sigma_{0 V}$ defines the BSC at normal incidence and $\sigma_{0}$ defines the BSC at a mid grazing angle. The dependence of BSC on grazing angle is depicted in Figure 3. 


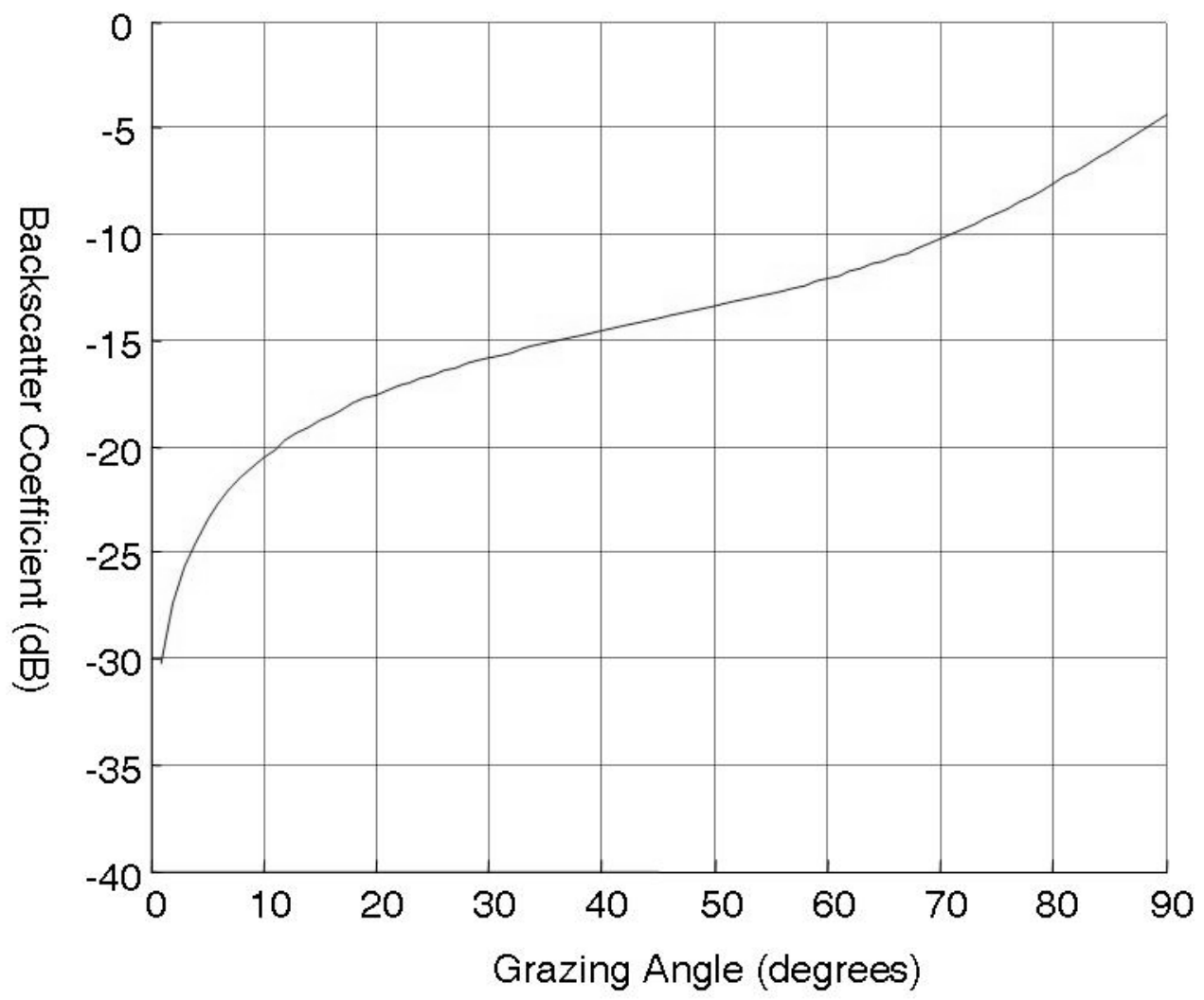

Figure 3: Surface Clutter Back Scatter Coefficient vs. Grazing Angle

The power of the clutter returns was calculated using the following form of the radar range equation in which the clutter RCS is cascaded with the transmitting and receiving antenna gains along the line of sight to the radar by reference to the appropriate antenna radiation pattern data.

$$
\text { clutter power }=\frac{P_{T} \cdot G_{T} \cdot G_{R} \cdot \lambda^{2} \cdot R C S_{C}}{64 \cdot \pi^{3} \cdot R_{S}^{4}}
$$

where $P_{T}=$ peak transmitted power $(=10 \mathrm{~kW})$

$$
G_{T}=\text { transmitting antenna gain }
$$




$$
\begin{aligned}
& G_{R}=\text { receiving antenna gain } \\
& R_{S}=\text { slant range } \\
& \lambda=\text { wavelength }=0.03 \mathrm{~m} \\
& R C S_{C}=\text { clutter radar cross section }=\mathrm{BSC} \times(x / y \text { resolution })^{2}
\end{aligned}
$$

The clutter power calculated from (2) was then range and Doppler gated and added into the appropriate range/Doppler cell on top of the noise power and any previously calculated clutter signals. PMC is applied by pre-calculating the Doppler gate of the centre of the main beam and applying this as an offset to the Doppler gated clutter. The total clutter and noise power is then stored in a two-dimensional matrix (range cell vs. Doppler cell) and displayed on a folded clutter map. Folded clutter maps in each PRF are derived which are subsequently required to produce the maps of target detectability. A folded clutter map is one in which the clutter amplitude from the full detection space of the radar is folded into one ambiguous range and Doppler interval. Thus a clutter map always has 64 equal intervals in Doppler, since this is the FFT size but a variable number of range cells which is equal to the number of range cells in one PRI. A constant fixed noise power level of $k \cdot T_{0} \cdot B_{n} \cdot F$ is included in every cell of the map, in which $k$ is Boltzmann's constant $=1.38 \times 10^{-23} \mathrm{~J} / \mathrm{K}, T_{0}$ is a standard temperature of $290 \mathrm{~K}, B_{n}$ is the noise bandwidth $=(\text { transmitted pulse width })^{-1}$ and $F$ is the noise figure $=3.16(5 \mathrm{~dB})$. An example of a folded clutter map is given in Figure 4 . The clutter map may be unfolded to cover the complete range/velocity detection space specified by the radar model by tiling the folded clutter map as many times as necessary. 


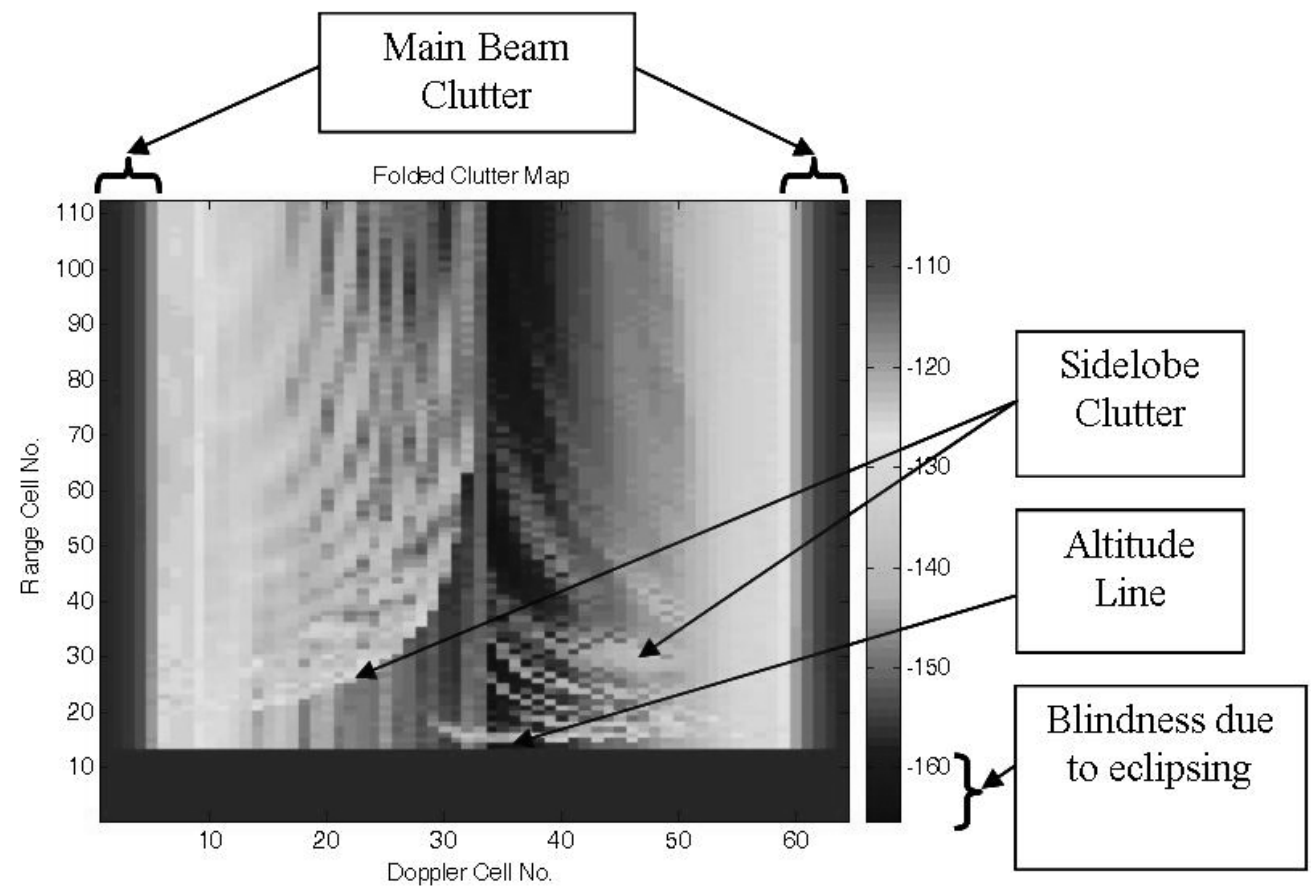

Figure 4: A Folded Clutter Map

$(P R I=56 \mu s)$

\subsection{Target detectability}

Target detectability over the full range/Doppler detection space of interest is conveniently represented by a detectability map [1]. The folded clutter map of Figure 4 is replicated in range and Doppler over the full detection space of interest (i.e. $185 \mathrm{~km}$ in range by $1500 \mathrm{~m} / \mathrm{s}$ in velocity) due to the repetition of data in the time and frequency domains. This results in an unfolded clutter map. Each PRF in the schedule has a similar, though different, unfolded clutter map. The probability of detection of a discrete target at any range/Doppler cell of interest depends on the number of PRFs in which the range/Doppler cell is not eclipsed and the probability of detection in each PRF, as determined by the SNCR of the cell. Blindness results from eclipsing, with no MBC blanking being assumed. A detectability map can therefore be derived over the 
full range and Doppler detection space of the radar and denotes the minimum target RCS required for detection at each range and Doppler cell in an appropriate number of PRFs. The detectability map may be thresholded at a given fixed RCS to indicate regions where a target of the given RCS would be visible/not visible. This thresholding forms the classic blind zone map for a medium PRF schedule. An example detectability map is given in Figure 5 based on a required $\mathrm{SNCR}=0 \mathrm{~dB}$ in at least three PRFs from the total of eight. Similar criteria have been used in the generation of all detectability maps used in this study. Should a more realistic SNCR of, for example, $+13 \mathrm{~dB}$ be required, one need only apply a $13 \mathrm{~dB}$ offset to the detectability map data. Therefore a target with RCS of $13 \mathrm{~dB}$ greater than the level read from the detectability map would be detected with a $P_{d}$ commensurate with the SNCR of $13 \mathrm{~dB}$.

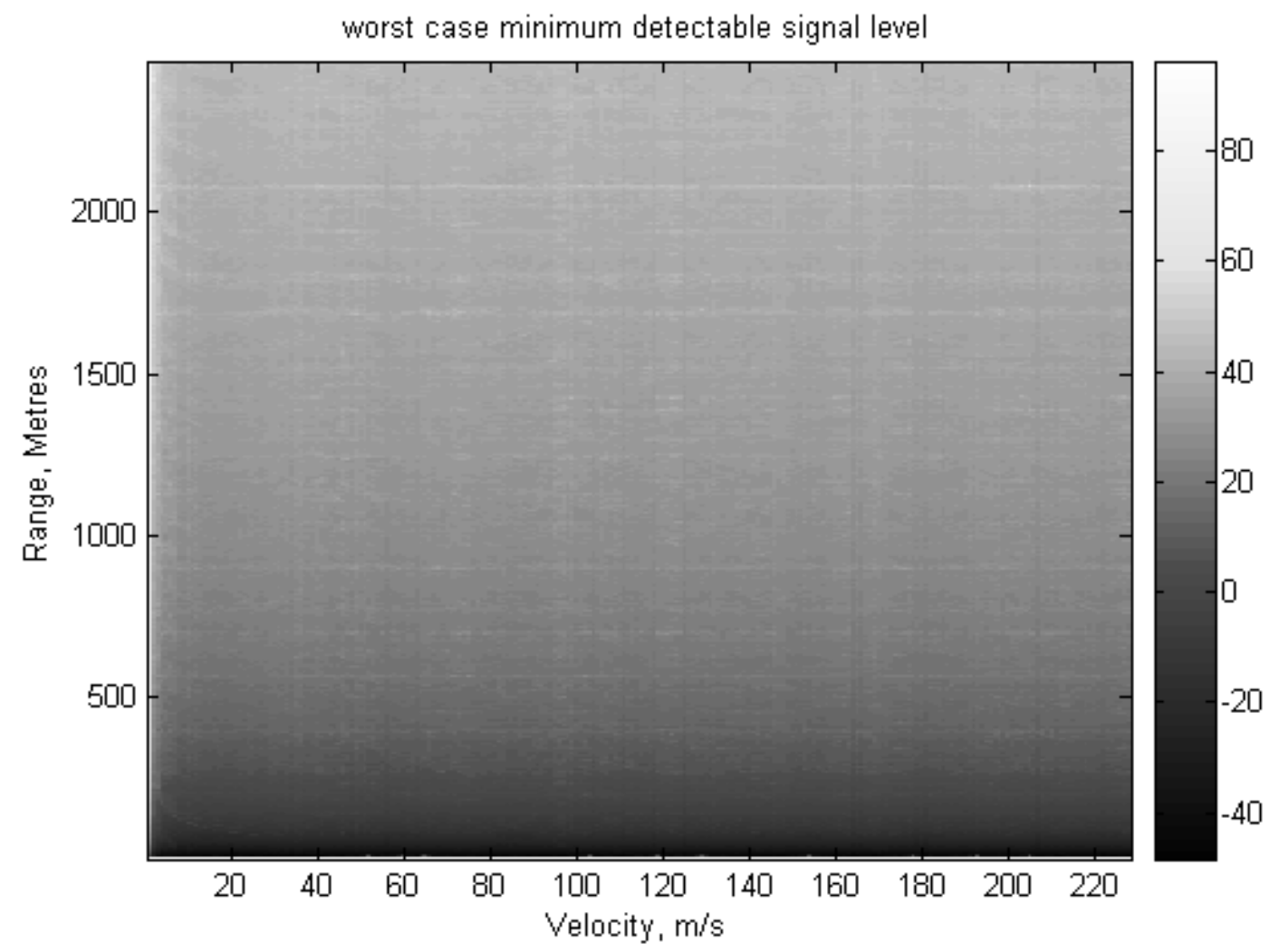

Figure 5: Detectability Map 
Whilst every effort has been maintained to ensure that the minimum target RCS requirement of the detectability maps is properly calibrated, there are inevitable sources of error. The radar range equation of (2) omits the integration gain and filter shape losses associated with the FFT process and also the atmospheric and system losses. Both static clutter and discrete targets would be subject to similar processing gains; subtle differences would arise depending on the temporal statistics of each, whereas losses affect both equally. For identical processing gains (clutter and discrete targets) the SCR would be independent of the processing gain. Integration gain has therefore been omitted from equation (2). As far as noise is concerned, the appropriate integration gain is applied in the generation of the detectability map. However, detection is generally clutter limited not noise limited. There is no distinction between clutter and discrete targets. There are no discrete targets within the (simulated) scene, just surface clutter. A detectability map simply plots the RCS that a target would have to possess if it were to be detected in the requisite number of PRFs ( 3 from 8 in this case) with a sufficient SCR (0dB in this case). This is the threshold value of RCS that triggers the transition from blindness to visibility in a classical blind zone map. The threshold RCS varies for each range/velocity cell within the detection space of the radar [1]. Without intimate knowledge of every aspect of the system design it would be impossible to calibrate the detectability maps. However, each detectability map is valid given the assumptions made in the radar, antenna and clutter models and therefore comparisons between detectability maps are also valid. Furthermore, comparisons remain valid irrespective of any offsets which may be applied to the detectability maps (such as may be required to depict a detection criterion of SNCR $\geq$ $+13 \mathrm{~dB}$ ) so long as a constant offset is applied to all detectability maps. In this work the benefits of different array weighting functions were derived through direct 
comparisons between detectability maps. Detectability maps are a useful means of characterizing relative performances in clutter.

\subsection{Evaluating target detectability}

Each of the 189 simulations results in eight folded clutter maps; one for each PRF. However, the resolution differs in each of the eight. A folded clutter map always has a fixed number of Doppler cells each of width $=P R F / F F T$ size, thereby fixing the number of Doppler cells to the FFT size (=64, in this case) but yielding a Doppler resolution which varies with PRF. The range cell width, however, is fixed by the compressed pulse width $(=0.5 \mu \mathrm{s}$ or $75 \mathrm{~m}$, in this case $)$ and so the number of range cells in the folded clutter map $=P R I / 0.5 \mu s$ and is therefore a function of the PRF. For an example PRF of $10 \mathrm{kHz}$ one obtains a folded clutter map of $64 \times 200=12800$ range/Doppler cells; each cell forming a pixel of the clutter map. For the case of a $10 \mathrm{kHz}$ PRF the transmitted pulse width is $10 \mu \mathrm{s}$ and so the number of blind (eclipsed) range cells $=21$. Thus the total number of blind range/Doppler cells is $21 \times 64=1344$ or $10.5 \%$ of the clutter map.

In constructing the detectability map, the eight folded clutter maps corresponding to the eight PRFs in the schedule are unfolded to occupy the whole range and Doppler detection space of the radar. This requires that they be read at a common resolution which can be no finer than that of the coarsest map. Hence the resolution of the detectability map is marginally coarser than the original clutter maps. The eight maps are then overlaid and the RCS level which toggles blindness is established. Since all detectability maps are plotted with a common resolution it is a simple exercise to 
compare two maps pixel by pixel since each pixel relates to a consistent range/Doppler cell. Due to the unfolding process, each detectability map is comprised of some 560000 pixels (range/Doppler cells).

In order to assess the dependence of target detectability on array weighting functions, a test strategy was developed in which the 27 detectability maps of one set of transmitting and receiving array weighting functions conditions (corresponding to the 27 combinations of azimuth/elevation steering angles, proportion of failed elements and platform altitude) were compared with the corresponding 27 detectability maps for each of the other six sets of transmitting and receiving array weighting functions. This progressed until all sets of transmitting and receiving array weighting functions had been compared with all the other sets. This test strategy has been found to be necessary due to the complexity of the optimization problem posed by this study. As an optimization problem, this work seeks to optimize a single objective; the detection performance of the radar, via the selection of an optimal combination of transmitting and receiving antenna weighting functions. However, the complexity arises because target detectability is quantified over several hundreds of thousands of range/Doppler cells (i.e. it is highly multi-dimensional) which must be distilled into simpler metrics. These optimization problems can typically yield several optimal solutions, collectively forming a Pareto optimal front [10], otherwise known as a trade-off surface. Optimal solutions identified by each set of comparisons may differ in each case depending on what metric is used to define target detectability and what baseline standard is adopted for each comparison. Two metrics $(X$ and $Y)$ have been derived which compare the data in pairs of detectability maps, $A$ and $B$. 
Both the $X$ and $Y$ metrics were derived to give a comparison of the detectability levels between two detectability maps. Since each detectability map comprises around 560000 pixels, the comparison of two maps is not trivial. The $X$ metric gives an impression of the relative area of the range/Doppler space for which the detectability of one test is greater than the detectability of another. The $X$ metric is related to the median of the differences between the two detectability maps. However, the $X$ metric is insufficient on its own to convey the general superiority of one map over another because it does not indicate the margin of any such superiority. Hence the $Y$ metric is also used. The $Y$ metric sums the differences (cubed) between the magnitudes of corresponding pixels of two detectability maps. The $Y$ metric is the third order moment of the difference data and is therefore representative of the skew in the distribution. The two metrics convey different aspects of the superiority of one map over another. The derivation of the two metrics is explained in the paragraphs below.

\section{(i) Ratio of comparisons, $X$}

The algorithm runs as follows:

- Exclude all the elements (i.e. Range-Doppler cell) of $A$ and $B$ which are in regions of eclipsed blindness. This avoids corrupting the statistical comparisons which follow.

- Derive a logical comparison matrix for which $A>B$ and sum all its elements. (The comparison matrix consists of elements $=1$ or 0 depending on whether $A>B$ or not. In summing all the elements one derives the total number of elements for which $A>B$.

- Derive a logical comparison matrix for which $B>A$ and sum all its elements. (The comparison matrix consists of elements $=1$ or 0 depending on whether $B>A$ or not. 
In summing all the elements one derives the total number of elements for which $B>A$.)

- These two sums do not necessarily sum to the total number of elements in the detectability map since the case of $A=B$ has not been computed. $A=B$ in blind regions and, elsewhere, $A=B=$ noise in regions of very low clutter.

- Derive the ratio of the two sums and express it on a decibel scale, i.e.:

$$
X=10 \cdot \log _{10}\left(\frac{\Sigma(A>B)}{\Sigma(B>A)}\right)
$$

If the detectability levels of $A$ are generally higher than those of $B$ then $\Sigma(A>B)$ is large and $\Sigma(B>A)$ is small, their ratio $>1$ and so $X$ is a (large) positive quantity. If the reverse is true then $X$ is a (large) negative quantity. The $X$ metric gives an impression of the relative area of the range/Doppler space for which the detectability of one test is greater than the detectability of another. The $X$ metric is related to the median of the differences between the two detectability maps. The $X$ metric is a first order statistic, similar to the mean, however, unlike the mean, it is less sensitive to highly skewed distributions. Regions of dominance of one over the other may be obtained by mapping the comparison matrices. The $X$ metric gives no information on the margin by which one is greater than the other.

\section{(ii) Sum of difference comparison, $Y$}

The algorithm runs as follows:

- Exclude all the elements of $A$ and $B$ which are in regions of eclipsed blindness. This avoids corrupting the statistical comparisons which follow. 
- Derive the matrix for $A-B$. This matrix yields signed difference values.

- Cube the difference matrix element by element. This accentuates the differences and preserves their sign.

- Sum all the elements of the cubed difference matrix. This returns the net difference over the whole range/Doppler detection space.

$$
Y=\Sigma(A-B)^{3}
$$

The $Y$ metric is the third order moment of the difference data and is therefore representative of the skew of the difference data distribution. If the detectability levels of $A$ are generally higher than those of $B$ then the $Y$ metric will be a (large) positive number whereas, if the reverse is true, the $Y$ metric will be a (large) negative number. The $Y$ metric gives an impression of the "aggregate" level by which the detectability of one test is greater than the detectability of another. However, one could not distinguish between the cases of a few elements in one matrix being significantly higher than those of the other matrix and most of the elements in one matrix being marginally higher than those of the other. Thus the $Y$ metric indicates the margin of superiority but not its extent in area.

The combination of the $X$ and $Y$ metrics therefore indicate both the area extent of superiority of one detectability map over another and also on the aggregate margin of this superiority. (Note: in all tests it was necessary to exclude the first column of the $A$ and $B$ matrices since these were dominated by main beam clutter and mask the subtle effects of the sidelobe clutter. This provides a partial filtering of $\mathrm{MBC}$ by excluding the very central region but retaining the peripheral data.) 
Of secondary interest is the effect of an increasing number of failed elements in the array. One expects failed elements to result in increased sidelobes and a reduction in mainbeam boresight gain and therefore a loss of detectability of targets in clutter, however, it is worth quantifying these changes in order to judge an acceptable number of failed elements. To evaluate this, it is necessary to make comparisons between detectability maps defined by a common patterns but differing in the proportion of failed elements, $P_{f e}$. As before, the detectability maps were compared using the same $X$ and $Y$ metrics previously described.

\section{Results \& Discussion}

\subsection{Array weighting function}

A series of comparisons has been made between pairs of detectability maps and the $X$ and $Y$ metrics of each comparison derived. Batches of 27 comparisons are made for each combination of transmitting and receiving array weighting functions and therefore the means $\bar{X}$ and $\bar{Y}$ over the 27 comparisons have been derived. A uniformly weighted mean has been used in this study, however, one might consider deriving a weighted mean in order to give preferential treatment of certain conditions e.g. zero steering angle. Note that the use of the mean of these metrics is not intended to imply that they have a Gaussian spread. Each detectability map has dimensions of $2467 \times 228=562476$. However, since the first column of each map (matrix) is excluded from the analysis, the processed data has dimensions $2467 \times 227=560009$. In some cases it is possible to obtain $\Sigma(B>A)=0$ and therefore an infinite value of $X$ 
results in which case the mean of $X$ would also be infinite. The maximum finite value of $X$ arises when $\Sigma(A>B)=560008$ and $\Sigma(B>A)=1$ and results in $X=57.48$. This is unlikely to arise since there exists the possibility that $A=B$ in some elements, however, some of the results approach to within 0.02 of this value. It was therefore decided to cap values of $X$ which would otherwise be infinite to a value of 57.50 . Similarly, values of $X$ which would otherwise be $-\infty$ are capped at -57.50 . In this way, the calculation of the mean is not confounded. The $\bar{X}$ results are given in Table 4 and the $\bar{Y}$ results in Table 5. Tables 4 and 5 offer mean results over a number of comparisons. The $A \_$patterns defines the transmit and receive weighting function used as a baseline against which all the other weighting functions are compared, as defined by the $B \_$patterns. Firstly, the $A \_$patterns is set to 1 (i.e. uniform on transmit and receive) and the $B \_$patterns is set to $2,3,4,5,6$ and 7 in turn. Next the A_patterns is set to 2 (uniform on transmit and Taylor $35 \mathrm{~dB}$ on receive) and the B_patterns is set to $1,3,4,5,6$ and 7 in turn. This continues with $A$ patterns being set to 3, 4, 5, 6 and 7 in their turn and for each value of $A \_$patterns, the $B \_$patterns cycles through all the other values. In this way, each combination of tranmit and receive weighting function is compared with all the other ones. (Actually, each is compared twice e.g. 1 vs. 3 and 3 vs. 1, hence the symmetrical/inverted nature of Tables 4 and 5.) This exhaustive method of comparison is necessary due to the high dimensionality of the test function; each detectability map being represented by about half a million pixels.

Both results tables are matrices with a leading diagonal of zeros and are symmetrical, and inverted about the leading diagonal. It is worth recalling that $X$ and $Y$ results which are positive mean that the minimum target RCS requirements defined by the $A$ 
detectability maps are greater than those of the $B$ detectability maps. Therefore, positive $X$ and $Y$ results across the $B \_$patterns rows denote the ability to detect smaller targets using the $B$ patterns when compared with the respective $A \_$patterns. The larger the positive results, the greater this margin. Negative results indicate the opposite.

\begin{tabular}{|c|c|c|c|c|c|c|c|c|}
\hline & & \multicolumn{7}{|c|}{ A patterns } \\
\hline & & 1 & 2 & 3 & 4 & 5 & 6 & 7 \\
\hline \multirow{7}{*}{ 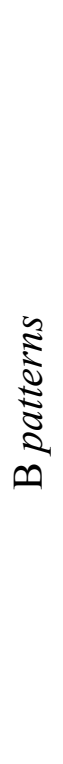 } & 1 & 0 & -49.8 & -51.8 & -45.9 & -47.1 & -40.6 & -44.0 \\
\hline & 2 & 49.8 & 0 & -7.2 & -13.7 & -15.7 & -7.7 & -11.5 \\
\hline & 3 & 51.8 & 7.2 & 0 & -5.8 & -12.5 & -0.4 & -6.1 \\
\hline & 4 & 45.9 & 13.7 & 5.8 & 0 & -5.5 & 5.7 & 0.4 \\
\hline & 5 & 47.1 & 15.7 & 12.5 & 5.5 & 0 & 8.8 & 5.1 \\
\hline & 6 & 40.6 & 7.7 & 0.4 & -5.7 & -8.8 & 0 & -6.0 \\
\hline & 7 & 44.0 & 11.5 & 6.1 & -0.4 & -5.1 & 6.0 & 0 \\
\hline
\end{tabular}

Table 4: $\bar{X}$ Results 


\begin{tabular}{|c|c|c|c|c|c|c|c|c|}
\hline & & \multicolumn{7}{|c|}{ A patterns } \\
\hline & & 1 & 2 & 3 & 4 & 5 & 6 & 7 \\
\hline \multirow{7}{*}{ 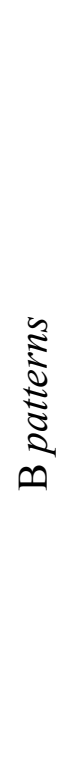 } & 1 & 0 & $-3 e 32$ & $-4 \mathrm{e} 32$ & $-5 e 32$ & $-5 e 32$ & $-6 e 32$ & $-6 \mathrm{e} 32$ \\
\hline & 2 & $3 e 32$ & 0 & $-3 e 29$ & $-6 . e 29$ & $-2 \mathrm{e} 30$ & $-3 e 30$ & $-5 e 30$ \\
\hline & 3 & $4 \mathrm{e} 32$ & $3 e 29$ & 0 & $-3 e 27$ & $-2 \mathrm{e} 29$ & $-4 \mathrm{e} 29$ & $-1 \mathrm{e} 30$ \\
\hline & 4 & $5 \mathrm{e} 32$ & $6 e 29$ & $3 e 27$ & 0 & $-9 \mathrm{e} 28$ & $-3 e 29$ & $-8 \mathrm{e} 29$ \\
\hline & 5 & $5 e 32$ & $2 \mathrm{e} 30$ & $2 \mathrm{e} 29$ & $9 \mathrm{e} 28$ & 0 & $-2 \mathrm{e} 28$ & $-1 \mathrm{e} 29$ \\
\hline & 6 & $6 e 32$ & $3 \mathrm{e} 30$ & $4 e 29$ & $3 \mathrm{e} 29$ & $2 \mathrm{e} 28$ & 0 & $-3 e 28$ \\
\hline & 7 & $6 e 32$ & $5 e 30$ & $1 \mathrm{e} 30$ & $8 \mathrm{e} 29$ & $1 \mathrm{e} 29$ & $3 \mathrm{e} 28$ & 0 \\
\hline
\end{tabular}

Table 5: $\bar{Y}$ Results

The rank order of the $B$ patterns from highest $\bar{Y}$ (best target detectability) to lowest $\bar{Y}$ (lowest target detectability) for all seven sets of results is consistently: 7, 6, 5, 4, 3, 2, 1. The consistency of this result is believed to be due to the simple arithmetic expression for $Y$. The rank order of the $B \_$patterns for the $\bar{X}$ results is not consistent across all seven sets of results. However, in all but one set of results i.e. those of A_patterns $=1$, the highest $\bar{X}$ (best target detectability) is obtained for $B \_$patterns $=5$. When $A \_$patterns $=1$, the highest $\bar{X}$ (best target detectability) is obtained for $B \_$patterns $=3$. In all seven sets of results the lowest $\bar{X}$ (worst target detectability) is consistently obtained for $B$ patterns $=1$ and it is obvious that patterns $=1$ is far removed from the optimal solution. The inconsistency in the rank order of the B_patterns for the $\bar{X}$ results when $A \_$patterns $=1$ is believed to be due to the fact that 
the baseline $\left(A \_\right.$patterns $\left.=1\right)$ is very distant from the better solutions. The small degree of inconsistency in the rank order of the patterns for the other $\bar{X}$ results is believed to be due to the non-arithmetical nature of the expression for $\bar{X}$. If one sums the $\bar{X}$ results over the seven sets of results $\left(\right.$ i.e. $\left.\sum \bar{X}\right)$ one obtains the rank order of the patterns from highest $\left(\sum \bar{x}\right)$ (best target detectability) to lowest $\left(\sum \bar{x}\right)$ (lowest target detectability) of: $5,4,7,3,6,2,1$.

The preferred solution depends on the metric used to quantify target detectability and the baseline against which comparisons are made. This is a typical dilemma associated with optimisation problems whose optimisation goal has high dimensionality and there is as yet no known metric which avoids the inconsistent behaviour observed here. One method to arrive at an optimum solution based on equal weightings of the $X$ and $Y$ results may be to award points to each of the patterns based on the rank order of their seven sets of $\bar{X}$ and $\bar{Y}$ results, i.e. a non-parametric normalization using rank ordering. The following scoring method is proposed here: 7 points are awarded for a first place in the rank order, 6 points for second place, 5 points for third and so on down to one point for a seventh place finish. Since also: $\operatorname{maximum} \sum \bar{X} \_$points $=\operatorname{maximum} \sum \bar{Y} \_$points $=49$, each patterns has an associated distance from the best possible solution given by:

$$
\text { DISTANCE }=\sqrt{\left(49-\sum \bar{X} \_ \text {points }\right)^{2}+\left(49-\sum \bar{Y} \_ \text {points }\right)^{2}}
$$

The points are displayed in Figure 6 in which "Best" indicates the maximum utopia solution. 
The ascending rank order of DISTANCE determines the rank order of the solutions defined by patterns. The results of the points scoring are (from best to worst): 7, 5, 6, $4,3,2$ and 1 .

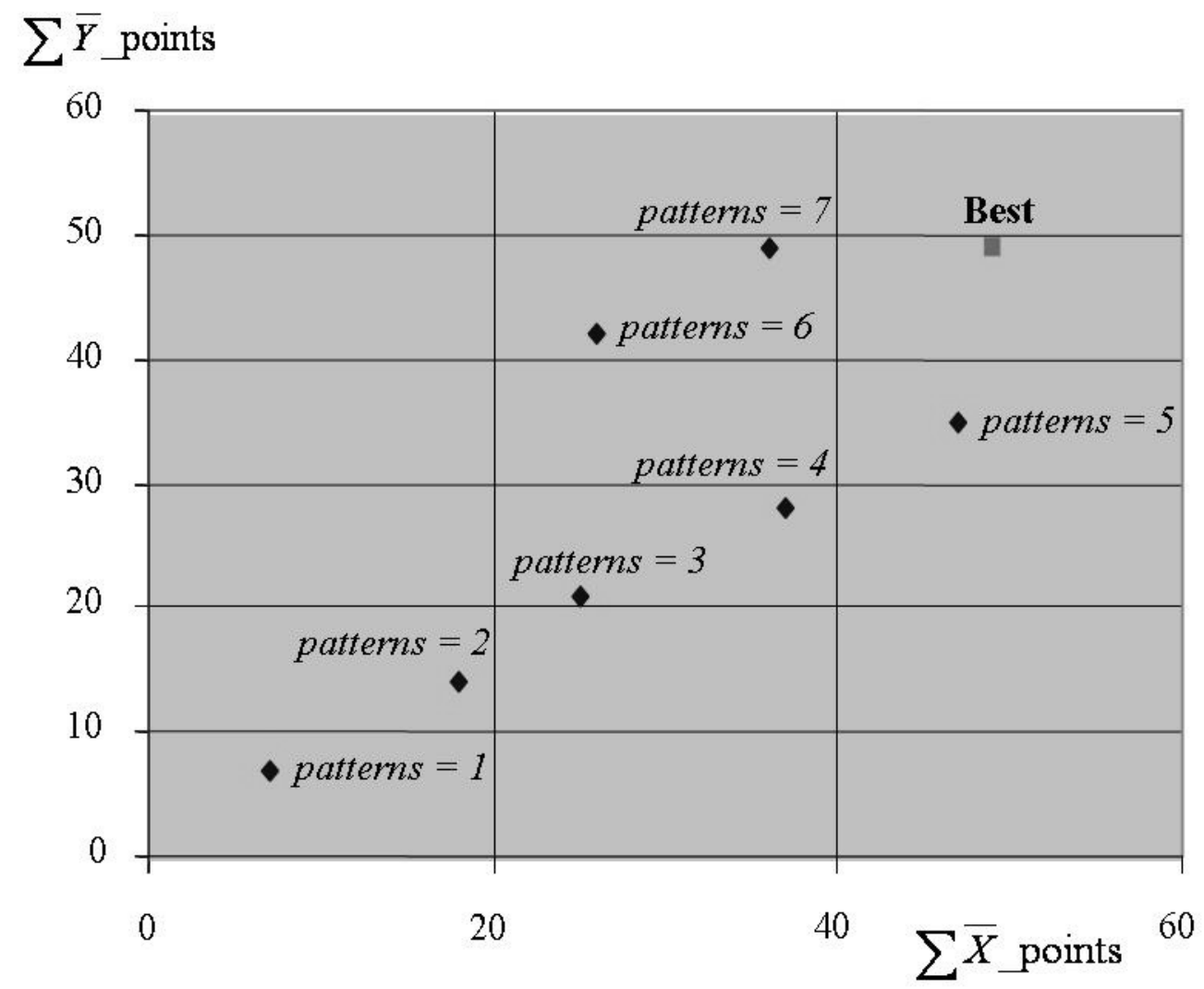

Figure 6: Points Positions of Solutions

From Figure 6 it is evident that patterns $=5$ and 7 are almost equal solutions which fall on a Pareto surface i.e. no one solution is better on both metrics simultaneously. However, of the two, patterns $=7$ offers the slightly better target detectability. Furthermore, it is believed that the rank order of the various results could alter if realistic statistical fluctuations were admitted in the various modelling procedures. It may be noted that the better target detectability is generally obtained for the Taylor 
$45 \mathrm{~dB}$ weighting function to be applied to the receiving array over the corresponding Taylor $35 \mathrm{~dB}$ function. The worst $X$ and $Y$ results were consistently obtained for patterns $=1$, indeed all solutions which entail the transmission using the Uniform weighting function (patterns $=1,2$ and 3) exhibit poor target detectability.

\subsection{Proportion of failed elements}

A further series of comparisons between detectability maps has been carried out to compare the cases of the probability of failed elements, $\mathrm{P}_{\mathrm{fe}}=0.00 \mathrm{vs} . \mathrm{P}_{\mathrm{fe}}=0.02$ and $\mathrm{P}_{\mathrm{fe}}=0.00$ vs. $\mathrm{P}_{\mathrm{fe}}=0.05$ for the various combinations of steering angles, altitude and transmitting and receiving array weighting functions. In this case, the $A$ detectability maps were taken to be those for $\mathrm{P}_{\mathrm{fe}}=0.00$, whereas the $B$ detectability maps were those of for which $\mathrm{P}_{\mathrm{fe}}=0.02$ and 0.05 . Thus there are two comparisons to be made $\left(\mathrm{P}_{\mathrm{fe}}=0\right.$ vs. $\mathrm{P}_{\mathrm{fe}}=0.02$ and $\mathrm{P}_{\mathrm{fe}}=0.00$ vs. $\left.\mathrm{P}_{\mathrm{fe}}=0.05\right)$ for the 9 different combinations of altitude, azimuth and elevation steering angles at each of the 7 combinations of transmitting and receiving array weighting functions (the 7 patterns). The results are given in Table 6 , in which the $X$ and $Y$ results have been averaged $(=\bar{X}$ and $\bar{Y}$, respectively) over all 7 patterns. As before, the values of $X$ which would otherwise be infinite have been capped to 57.50 and the use of the means in Table 6 is not intended to imply that the metrics have a Gaussian spread.

\begin{tabular}{|c|c|c|c|c|c|c|}
\hline $\mathrm{P}_{\mathrm{fe}}(\mathrm{A})$ & $\mathrm{P}_{\mathrm{fe}}(\mathrm{B})$ & Azimuth & Elevation & Altitude & $\bar{X}$ results & $\bar{Y}$ results \\
& & steering & steering & $(\mathrm{m})$ & & \\
& & angle & angle & & & \\
& & $(\mathrm{deg})$ & $(\mathrm{deg})$ & & & \\
\hline
\end{tabular}




\begin{tabular}{|c|c|c|c|c|c|c|}
\hline 0.00 & 0.02 & 0 & 0 & 5000 & -19.5 & $-3 e 21$ \\
\hline 0.00 & 0.05 & 0 & 0 & 5000 & -38.6 & $-2 \mathrm{e} 22$ \\
\hline 0.00 & 0.02 & 30 & 0 & 5000 & -18.0 & $1 \mathrm{e} 22$ \\
\hline 0.00 & 0.05 & 30 & 0 & 5000 & -21.9 & $3 e 23$ \\
\hline 0.00 & 0.02 & 56 & 0 & 5000 & -14.1 & $-1 \mathrm{e} 20$ \\
\hline 0.00 & 0.05 & 56 & 0 & 5000 & -19.2 & $-2 \mathrm{e} 22$ \\
\hline 0.00 & 0.02 & 0 & -5.5 & 5000 & -20.9 & $3 \mathrm{e} 24$ \\
\hline 0.00 & 0.05 & 0 & -5.5 & 5000 & -32.2 & $9 \mathrm{e} 25$ \\
\hline 0.00 & 0.02 & 30 & -5.5 & 5000 & -14.2 & $2 \mathrm{e} 29$ \\
\hline 0.00 & 0.05 & 30 & -5.5 & 5000 & -19.6 & $2 \mathrm{e} 30$ \\
\hline 0.00 & 0.02 & 56 & -5.5 & 5000 & -12.4 & $3 \mathrm{e} 29$ \\
\hline 0.00 & 0.05 & 56 & -5.5 & 5000 & -13.9 & $2 \mathrm{e} 30$ \\
\hline 0.00 & 0.02 & 0 & 0 & 1000 & -17.4 & $-7 e 26$ \\
\hline 0.00 & 0.05 & 0 & 0 & 1000 & -33.7 & $-9 e 27$ \\
\hline 0.00 & 0.02 & 30 & 0 & 1000 & -13.9 & $1 \mathrm{e} 29$ \\
\hline 0.00 & 0.05 & 30 & 0 & 1000 & -17.6 & $3 \mathrm{e} 30$ \\
\hline 0.00 & 0.02 & 56 & 0 & 1000 & -9.4 & $6 \mathrm{e} 28$ \\
\hline 0.00 & 0.05 & 56 & 0 & 1000 & -14.4 & $7 \mathrm{e} 30$ \\
\hline
\end{tabular}

Table 6: $\bar{X}$ and $\bar{Y}$ vs. $\mathrm{P}_{\text {fe }}$ results 
All the $\bar{X}$ metric values are negative indicating that detectability levels increase (i.e. targets need to be larger to be detected) for $\mathrm{P}_{\mathrm{fe}}>0.00$, which is unsurprising. The larger negative magnitude of $\bar{X}$ is consistently obtained for $\mathrm{P}_{\mathrm{fe}}(\mathrm{B})=0.05$, as opposed to $\mathrm{P}_{\mathrm{fe}}(\mathrm{B})=0.02$, which, again, is to be expected. Comparing the cases of zero element failures to $2 \%$ element failures results in $\bar{X}$ ranging from -20.9 to -9.4 , mean $=-15.5$, which is comparable to the difference in target detectability between patterns 2 (Uniform on transmit and Taylor $35 \mathrm{~dB}$ on receive) and patterns 5 (RTT on transmit and Taylor $45 \mathrm{~dB}$ on receive), see Table 4 . Comparing the cases of zero element failures to $5 \%$ element failures results in $\bar{X}$ ranging from -38.6 to -14.4 , mean $=-$ 23.5. In this case there is no near comparison with the margins in target detectability between the combinations of patterns from Table 4 . Nevertheless, it is clear that the effects of $2 \%$ and $5 \%$ element failures is comparable to some of the more significant differences in target detectability between some of the best and worst array weighting functions, as quantified by the ratio of comparisons metric. The $\bar{Y}$ metrics vary between positive and negative values for different combinations of conditions, there are no consistent trends and the $\bar{Y}$ metric data is rather inconclusive. However, the magnitudes of $\bar{Y}$ are typically several orders of magnitude lower than those of Table 5, section 3.1, indicating typically smaller margins of superiority/inferiority in detectability performance. The changes in target detectability due to 2 and $5 \%$ failed elements are, on the whole, less significant than the effects of array weighting functions. The combination of the two metrics suggests that failed elements do result in large regions of the range/velocity detection space of the radar where target detectability has degraded. However, over the whole range/velocity detection space the aggregate margin by which target detectability changes is small and inconsistent. Therefore, the regions of degraded target detectability exhibit relatively small margins 
of degradation. It is also reasonable to assume that partial failures of elements would result in smaller regions and margins of degraded target detectability.

\subsection{Sensitivity of results to conditions}

The data of Tables 4, 5 and 6 and the scales of Figure 6 are difficult to calibrate. It is difficult to derive any absolute level of performance and these Tables and Figures only yield comparative performances. It is in the nature of the problem that the margins of one scenario over another cannot be reduced to a single figure; it is not possible to claim that target detectability in one scenario is $x \mathrm{~dB}$ better than another because of the variation across the scene. This study is based on a sample of typical combinations of realistic operating conditions which the authors believe to be representative of most clutter limited situations and hence the conclusions drawn from these results are valid in this context.

With regard to the sensitivity of the results to the model parameters, some comments on each of these is offered below:

Distortion of the beam patterns. It can be seen that the margins between patterns 5 and 7 is marginal. Clearly, the difference between these two cases is so small that it would probably be masked by noise, clutter statistical variation and target fluctuation. Therefore, it is quite clear that target detectability is not particularly sensitive to small variations in beam pattern.

Clutter. All statistical variation in clutter has been deliberately eliminated as it was feared that such random variations might mask small changes due to the array 
weighting functions. Given the near identical performances of patterns 5 and 7, this seems to have been justified since the introduction of clutter statistics would probably cloud the judgement between similar beam patterns; it would introduce another, unwanted and unknown, factor. Variations in clutter statistics have been considered in $[1]$.

Failure Probabilities. Three probabilities of failure have been considered. The results have been presented and discussed and enable the reader to make comparisons between these three cases. These results have also been compared to the differences due to the use of differing array weighting functions. In the ensuing discussion it was noted that the $X$ and $Y$ metrics did not reveal consistent trends but that in the worst case (i.e. the $X$ metric) the effects of $2 \%$ and $5 \%$ element failures is comparable to some of the more significant differences in target detectability between some of the best and worst array weighting functions. However, more typically, the consequences of up to $5 \%$ element failures on target detectability are relatively small and inconsistent.

PRFs. Previous work concludes that target detectability is highly sensitive to the exact choice of PRF values, number of PRFs used and criterion for detection (e.g. 3 of 8). This is an important subject area and has been the subject of much of the authors' previous work extending back over several years and is reported on in references [1] [5]. The techniques reported on in these references have been used to derive a nearoptimum PRF set for the radar assumed in this study. 
Blindness. The sensitivity of target detectability to eclipsing blindness has not specifically been considered in this work, however, the blindness problem has been considered along side the authors' earlier work on the exact choice of PRF values, number of PRFs used and criterion for detection (e.g. 3 of 8), in references [1] - [5].

In summary, there is no simple metric to relate the sensitivity of results to other parameters. These issues are far from trivial, however, several of these have been major research topics in their own right and have been reported on in previous papers.

\section{Conclusions}

Clearly, the differing metrics which one may use to quantify target detectability result in differing solutions with very little to chose between them. However, by combining the means of both the $X$ and $Y$ metrics in a points scoring system the best overall solution was identified as being the combination of the SPTN function on transmission and the Taylor $45 \mathrm{~dB}$ function on reception. This was very closely followed by the combination of the RTT function on transmission and the Taylor $45 \mathrm{~dB}$ function on reception. The overall preference for the former may well be due to its lower RMS sidelobe levels in the lower hemisphere. Nevertheless, it ought to be stressed that the margins between these two cases are very small and may very well be masked by statistical variations in noise, clutter and target RCS. It may also be worth noting that the RTT function results in an effective radiated power (ERP) some $0.6 \mathrm{~dB}$ higher than that of the SPTN function and so enjoys a small advantage in detection performance in noise limited cases. Furthermore, the RTT function (and its resulting beam pattern) is circularly symmetrical and so remains constant irrespective of the platform roll angle. The worst target detection performance was obtained when 
using the Uniform weighting function on the transmitting array. Indeed the test case of the Uniform function on both transmission and reception was found by both metrics to yield the worst target detection capability by a large margin.

Target detectability degraded as the proportion of failed elements increased from zero to $5 \%$. Failure of the elements contributes towards increases in the sidelobes, reduction in mainbeam boresight gain and hence the reduction in detection performance. Failed elements result in significant regions of the range/velocity detection space of degraded target detectability, however, the margins by which detectability is degraded tended to be less than the margins between detectability using the best and worst array weighting functions. A failure of $5 \%$ of the array elements resulted in modest, though meaningful, degradations in target detectability. Therefore, 5\% would be an appropriate upper limit on the proportion of failed elements.

These conclusions pertain to a reasonable sample of operating scenarios which were designed to result in clutter limited detection conditions for medium PRF operation. The authors believe that these conclusions remain valid for different, though similar, scenarios resulting in clutter limited detection conditions. Should the radar operate at substantially higher altitudes and/or in look-up attitudes and/or in high PRF modes, then detection is quite likely to become noise limited. Noise limited detection conditions will result in different solutions for optimal PRF values, FFT sizes and numbers of coherent processing intervals and may also lead to different solutions for optimal array weighting functions. 


\section{Acknowledgments}

This work was carried out as part of the UK MOD funded Output 3 Research Programme.

The array antenna data was supplied by QinetiQ, Malvern. 


\section{References}

[1] C. M. Alabaster \& E. J. Hughes. "The Design of Medium PRF Radar Schedules For Optimum Detectability in Diverse Clutter Scenes" Proc. IEEE Waveform Diversity \& Design, Lihue, Kaua'i, HI, USA, $22^{\text {nd }}-27^{\text {th }}$ January 2006.

[2] C.M. Alabaster \& E.J. Hughes "Novel PRF Schedules for Medium PRF Radar", Proc. Radar 2003, Adelaide, 3rd - 5th Sept. 2003.

[3] C.M. Alabaster, E.J. Hughes \& J.H. Matthew. "Medium PRF radar PRF Selection Using Evolutionary Algorithms", IEEE Trans. Aerospace and Electronic Systems, vol. 39, no. 3, pp. 990-1001, July 2003.

[4] P. G. Davies and E. J. Hughes. "Medium PRF set selection using evolutionary algorithms", IEEE Transactions on Aerospace and Electronic Systems, vol. 38, no. 3, pp.933-939, July 2002.

[5] D. A. Wiley, S. M. Parry, C.M. Alabaster and E. J. Hughes. "Performance Comparison of PRF Schedules for Medium PRF Radar”, IEEE Trans. Aerospace and Electronic Systems. vol. 42, no. 2, Apr 2006, pp 601-611.

[6] C. M. Alabaster \& E. J. Hughes. "The Dependence of Radar Target Detectability on Array Weighting Function" Proc. Radar 2007, Edinburgh, UK, $15^{\text {th }}-18^{\text {th }}$ October 2007.

[7] S. C. Iglehart, "Design of Weighting Functions Under Peak Amplitude and Effective Radiated Voltage Constraints.”, IEEE Transactions on Aerospace and Electronic Systems., Vol 15, No.2, pp. 267-274, March 1979.

[8] I. M. Mellor, F. J. Adams. "Benefits of Transmit Beam Pattern Synthesis for Airborne Phased Array Radar", Proc. IRS 2005, Berlin, Germany, 6 - 8 Sept. 2005. 
[9] G. Poulton, "Power pattern synthesis using the method of successive projections.”, Proc. Antennas and Propagation Society /international Symposium, pp. 667-670, 1986.

[10] Kalyanmoy Deb, Multi-objective optimization using evolutionary algorithms, John Wiley \& Sons, 2001, ISBN 0-471-87339-X. 
Author's Name: Clive M. Alabaster

Author's Affiliation: Cranfield University

Shrivenham, UK

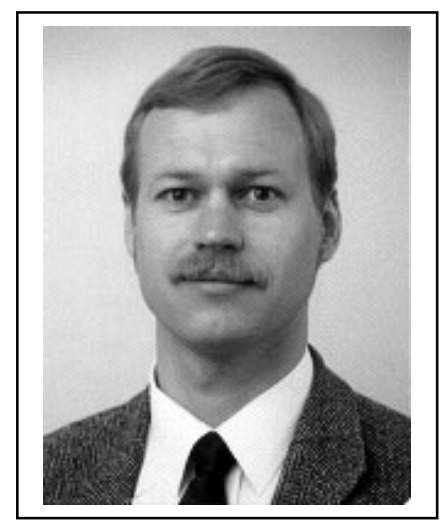

Dr. Clive M. Alabaster received his BSc degree in Physics with Microelectronics from University College Swansea, Wales, in 1985 and his $\mathrm{PhD}$ from Cranfield University, Shrivenham in 2004. From 1985 to 1992 he worked as a microwave design and development engineer on airborne radar systems with GEC Marconi, Milton Keynes, England. From 1992 to 1998 he worked as a lecturer in radar techniques at Arborfield Garrison, near Reading, England. He joined Cranfield University, Shrivenham, UK in 1998 as a lecturer and now works in the Sensors group within the Department of Informatics and Sensors. His research interests include the pulse Doppler radar, radar waveform design and the dielectric properties of materials, particularly in the millimetre wave band. He is a member of the Institute of Physics and is a Chartered Engineer.

Email: c.m.alabaster@cranfield.ac.uk 
Author's Name: Evan J. Hughes

Author's Affiliation: Cranfield University

Shrivenham, UK

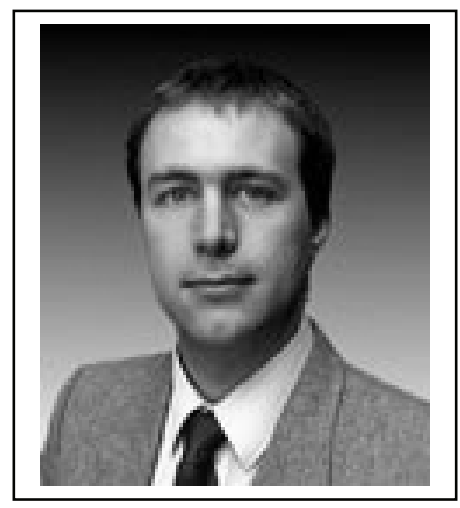

Dr. Evan J. Hughes received his BEng and MEng degrees in Electrical and Electronic Engineering from the University of Bradford, England, in 1993 and 1994 respectively. From 1993 to 1995 he worked as a design engineer with GEC Marconi, Leicester. He received his Ph.D. in 1998 from Cranfield University, Shrivenham, England. His primary research interests include noisy multi-objective evolutionary algorithms, swarm guidance, data fusion, artificial neural networks, fuzzy systems, intelligent agents and radar systems. He is a member of both the IEE and the IEEE and is a Chartered Engineer. He received the prize for best paper at GALESIA '97, Glasgow, UK, won the Evolutionary Checkers Competitions at CEC 2001, Seoul, S. Korea and WCCI 2002, Honolulu, Hawaii, and won the Time Series Prediction Competition at WCCI 2002. He is currently working as a senior lecturer for the Sensors group in the Department of Informatics and Sensors.

Email: ejhughes@theiet.org 